\title{
WIUTE P因P展圆
}

\section{Self-Pinched Beam Transport Experiments Relevant to Heavy Ion Driven Inertial Fusion Energy}

\author{
January 30, 1998
}

W. B. Herrmannsfeldt

Stanford Linear Accelerator Center, Stanford, CA 94309

R. O. Bangerter, T. J. Fessenden, E. P. Lee, S. S. Yu Lawrence Berkeley National Laboratory. Berkeley, CA 94720

C. L. Olson

Sandia National Laboratories, Albuquerque, NM 87185

D. R. Welch

Mission Research Corporation, Albuquerque, NM 87106

J. J. Barnard, A. Friedman, B. G. Logan, R. W. Moir

Lawrence Livermore National Laboratory, Livermore, CA 94551

I. Haber, P. F. Ottinger, F. C. Young

Naval Research Laboratory, Washington, DC 20375

R. R. Peterson University of Wisconsin, Madison, WI 53706

R. J. Briggs

Science Applications International Corporation, Pleasanton, CA 94588 


\section{Introduction}

An attractive feature of the inertial fusion energy (IFE) approach to commercial energy production is that the fusion driver is well separated from the fusion confinement chamber. This "standoff" feature means the driver is largely isolated from fusion reaction products. Further, inertial confinement fusion (ICF) target ignition (with modest gain) is now scheduled to be demonstrated at the National Ignition Facility (NIF) using a laser driver system. The NIF program will, to a considerable extent, validate indirectly-driven heavy-ion fusion (HIF) target designs for IFE. However, it remains that HIF standoff between the final focus system and the fusion target needs to be seriously addressed. In fact, there now exists a timely opportunity for the Office of Fusion Energy Science (OFES) to experimentally explore the feasibility of one of the attractive final transport options in the fusion chamber: the self-pinched transport mode.

Presently, there are several mainline approaches for HIF beam transport and neutralization in the fusion chamber ${ }^{1,5^{*}}$. These range from the (conservative) vacuum ballistic focus, for which there is much experience from high energy research accelerators, to highly neutralized ballistic focus, which matches well to lower voltage acceleration with resulting lower driver costs. Alternatively, Z-discharge channel transport and self-pinched transport in gas-filled chambers may relax requirements on beam quality and final focusing systems, leading to even lower driver cost. In any case, these alternative methods of transport, especially self-pinched transport, are unusually attractive from the standpoint of chamber design and neutronics. There is no requirement for low chamber pressure. Moreover, only a minuscule fraction of the fusion neutrons can escape from the chamber. Therefore, it is relatively easy to shield sensitive components, e.g., superconducting magnets from any significant neutron flux. Indeed, self-pinched transport and liquid wall protection endow DT fusion with many of the advantages of aneutronic fusion. The question is: will self-pinched transport work?

Early theoretical studies ${ }^{\prime}$ indicated that self-pinched transport was not an option because net currents established in gas during beam injection were too small to cause beam pinching. However, recent numerical simulations using the 3D hybrid code IPROP ${ }^{3}$, including the effects of non-local ionization, indicate that self-pinched transport may be possible. The capability to test the concept exists today in scaled experiments using a highcurrent focused proton beam produced by the Gamble II pulsed-power accelerator at the Naval Research Laboratory. This White Paper describes the implications of the selfpinched transport approach to $\mathrm{HIF}$ power plant design and the relevance of proton experiments designed to test the concept. Near-term experiments and analysis are also suggested.

* References are grouped into five categories in an appendix. 


\section{A Survey of Final Transport Options}

Typically, final-focus/final transport design ${ }^{5}$ employs large-aperture magnetic lenses located close $(\leq 5 \mathrm{~m})$ to the fusion chamber, which directly focus large $(\approx 15 \mathrm{~cm})$ diameter beams onto the fusion target. The required small beam radius $(\leq 5 \mathrm{~mm})$ is reached only in the vicinity of the target. This scenario is essentially an application of charged particle optics of the conventional type although modified by space charge, ambient gas, and introduced electrons for neutralization. The major alternative is to focus the beams to small radius outside the fusion chamber (or at its edge) and arrange conditions within the chamber so that the beams propagate with small radius to the target ${ }^{4}$. Inside the chamber this requires both electrical neutralization by plasma electrons and a sufficiently large forward current flowing within each beam to provide effective magnetic confinement of the high energy ions. Z-discharge channel transport and self-pinched transport are examples of this alternative type of beam transport to the target.

\section{Self-Pinched Transport Option}

Plasma electrons are generated largely by the interaction of the ion beams with background gas, which also strips the beam ions to a high charge state (up to $q=55$ for Cs). Electrical curtent is provided by the stripped ion beam itself in the self-pinched propagation mode; however, this current is expected to be partially canceled by a comoving induced electron current. In this respect, self-pinched transport differs significantly from Z-discharge-channel transport, where an azimuthal magnetic field is frozen into the plasma along the desired path to the target prior to ion beam injection. A typical example ${ }^{4}$ of self-pinched beam parameters starts with 16-24 beams of $7.5 \mathrm{kA} \mathrm{Cs}^{+}$, each focused to a 2-5 $\mathrm{mm}$ radius spot on a stripping foil or gas puff near the fusion chamber entrance (Figure 1). After stripping, the ion current is about $400 \mathrm{kA}$ per beam, but with an approximately equal negative current of co-flowing electrons. One of the main issues of pinched transport of ion beams is the extent to which the electron current flows at larger radius than that of the beams, and thereby allows a net pinch force to be maintained. As little as $10 \mathrm{kA}$ net current (beam and electrons) inside the ion beam radius may be sufficient for propagation. Obtaining even this low effective value of current would be problematic if the electron current could be attributed purely to inductive drag in a plasma with scalar conductivity generated locally by the beam. However, recent results with the IPROP $^{3}$ code have indicated that a large fraction (up to $50 \%$ ) of the electron current is indeed spread to larger radius by non-ohmic effects from beam-generated energetic secondary electrons and an expanded beam head characteristic of propagating ion beams.

It is clear that pinched propagation, if feasible, offers significant advantages for the fusion chamber design. Among these are the elimination of the large aperture beam ports which thereby greatly reduces the flux of fusion products into the beam lines, simplified final focus magnets, and a much reduced vacuum requirement (from $10^{-3}$ Torr up to $\approx 10^{-1}$ Torr). Further attractive features for the total fusion driver result if pinched propagation is employed for the several hundred meters of drift compression upstream of the fusion chamber (called the mini-focus concept). This extended self-pinched concept drastically reduces the size and complexity of the final focus magnet arrays and also allows a large increase in beam momentum spread in the accelerator (possibly decreasing driver cost and improving beam stability). 


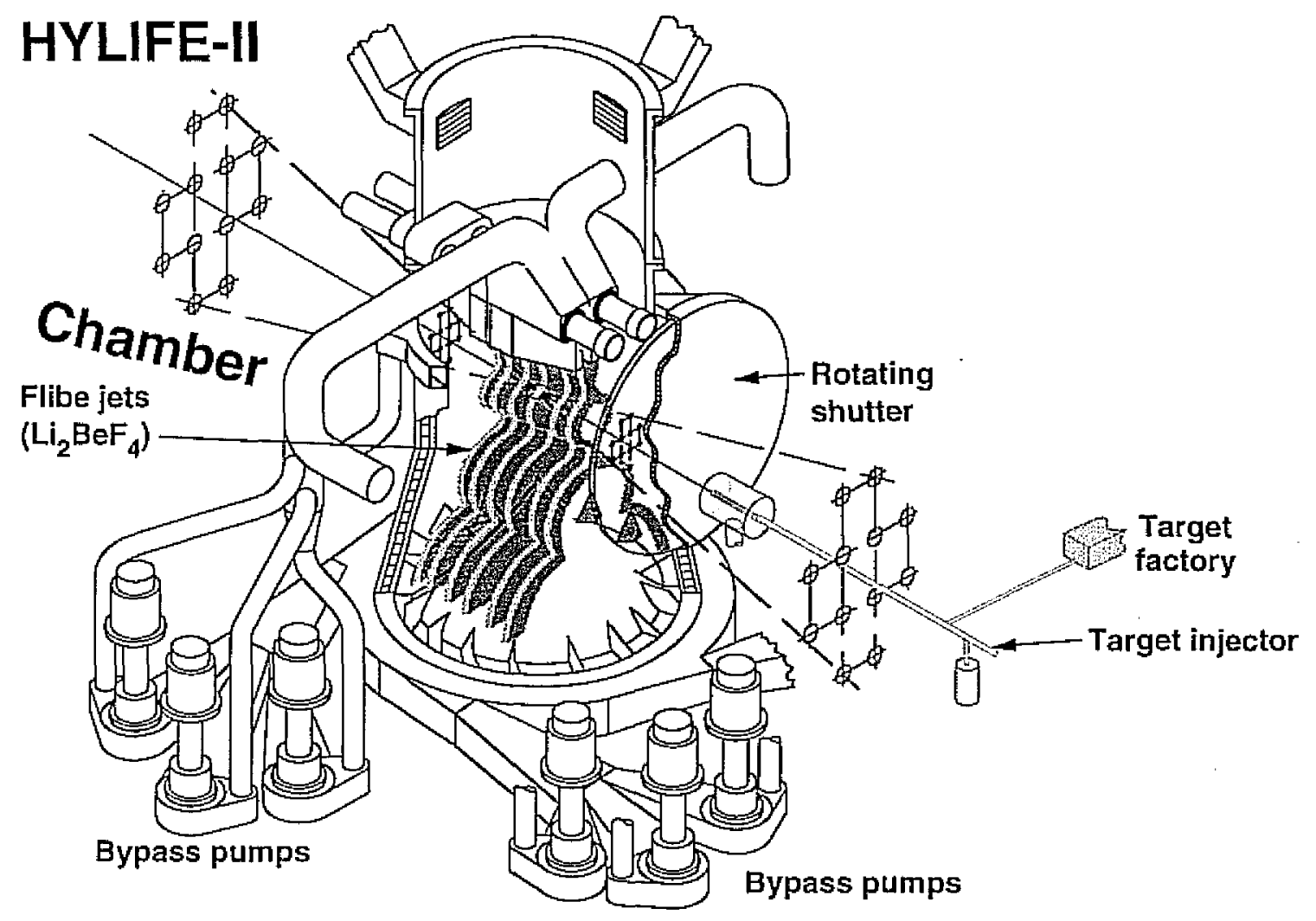

Figure 1

This HYLIFE II Chamber (5) accommodates 24 conventionally focused beams. These could be replaced by self-pinched beams on similar trajectories, and the large rotating shutters would be removed.

\section{Z-Discharge-Channel Transport Option}

Studies of a closely-related final-focus/chamber transport concept are currently in progress at LBNL. This concept supplies the azimuthal magnetic field needed to confine the beam by using an externally generated Z-discharge that is established before the heavy ion beam arrives. The Z-discharge field guides the heavy ion beam through the reactor chamber to the fusion target. The current-carrying channels are formed using a laser to generate a linear reduced density channel that connects the beam input port to the fusion target. At the proper moment, a high voltage pulse forms a plasma discharge along the channel with currents of 50 to $100 \mathrm{kA}$, to focus and guide the heavy ion beam to the target. Perhaps the major physics risk in this concept lies in establishing and controlling the current-carrying channels. A disadvantage is the addition of the laser that forms the channel and the addition of electrodes, insulators, and pulse power circuitry to the inertial fusion chamber.

A schematic of an inertial fusion reactor concept is shown in Figure 2, which is representative of a $Z$-discharge target chamber. An adiabatic lens focuses several incoming beams of heavy ions into a $Z$-discharge-channel that guides and transports the combined beams to the fusion target.

Advantages of the Z-discharge chamber scheme include the nearly complete wall beam entry port protection with thick Flibe (F-Li-Be)jets (except for several small holes), protection of final superconducting magnets with water-cooled stainless steel, and relaxed 
emittance and energy spread requirements on the heavy ion beam; these also carry over to the self-pinched scheme.

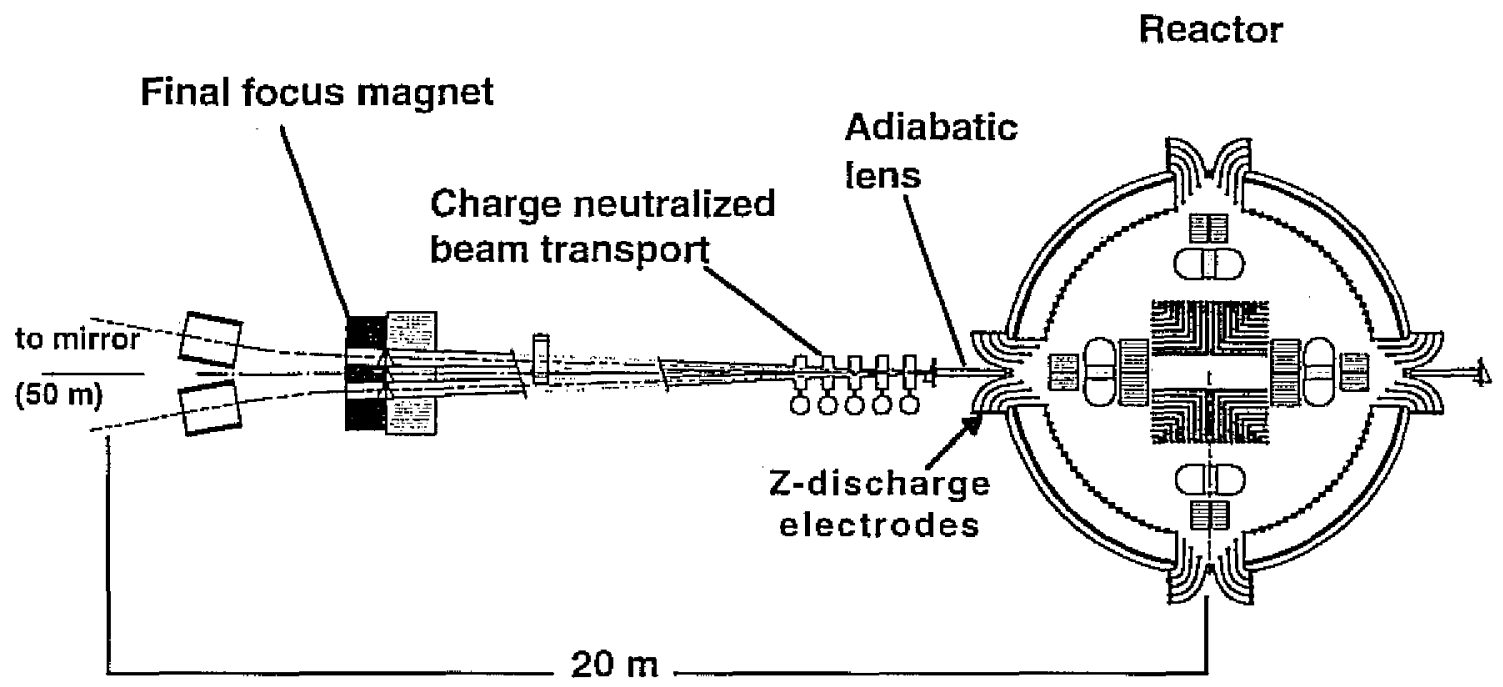

Figure 2

\begin{abstract}
Z-discharge power plant concept showing final quadrupole focus magnets, charge neutralized beam transport before final focus through an adiabatic plasma lens, and channel transport through the reactor to the target. Flibe jets in the chamber are illustrated.
\end{abstract}

Neutralized Ballistic Transport Options

The conservative vacuum ballistic focus with quadrupole final focus magnet sets, for which there is much applicable experience in high energy accelerators, is well matched to the high energy (10 GeV) range of accelerator options. However, lower ion energy is expected to lead to lower driver cost. Programmed injection of cold electrons into a ballistically focused beam neutralizes space charge, and therefore permits higher currents, lower kinetic energy and lower ion mass; this reduces driver cost but with an increase in physics uncertainty in the chamber. It may also be used for aberration correction, and offers the possibility of control of beam spots to match the requirements of distributed radiator targets. Recent PIC calculations' suggest the feasibility of using modest currents of low energy electrons to modify the radial electric field profiles within the ion beam. Electron injection could be done many different ways, but manageable currents of injected electron beams, if properly programmed spatially and temporally, could also provide a means of correcting geometric aberrations and also make dynamic chromatic aberration corrections for velocity variations with time. This, in turn, could make it possible to do neutralized drift compression to keep beam electrostatic potentials manageably small. In summary, the ballistic options may be able to match some of the advantages of self-pinched transport as far as beam quality and driver costs are concerned. It is, however, unlikely 
that they will be as advantageous as the self-pinched mode in terms of chamber design and neutronics.

\section{Relevance of Proton Experiments to Heavy Ion Fusion IFE}

Several final transport schemes for heavy ion driven IFE use beam transport in a gas, or in a current-carrying discharge formed in an initially-neutral gas. These schemes include ballistic transport in neutral gas, Z-discharge-channel transport, and self-pinched transport. Typically, "vacuum transport" occurs at pressures less than $10^{-4}$ Torr lithium; stripping of a heavy ion beam with charge state $\mathrm{q}=+1$ to $\mathrm{q}=+2$ is well underway at $10^{-3}$ Torr lithium; rapid stripping to high charge states occurs at $10^{-2}$ Tor lithium; and essentially full stripping occurs for pressures above about $10^{-1}$ Torr lithium. Although the injected heavy ion beam (particle) current for IFE may be only $10 \mathrm{kA}$ per beam, when fully-stripped the heavy ion beam electrical current $I_{e}$ can approach 1.0 MA. For these high electrical currents, charge and current neutralization must be accurately controlled to assure successful beam propagation. To study these phenomena experimentally requires the use of high current beams. Presently, there are no high current (10 kA or more) heavy ion beams anywhere in the world. However, there are high-current (100 kA or more) proton beams that could be used to model the heavy ion beam transport processes.

Specifically, for self-pinched transport, a comparison can be made of the net currents required for heavy ion fusion IFE to those required for a proton experiment. Consider an ion beam of atomic mass number $A$, atomic number $Z$, and charge state $q$ leaving a final focus magnet system with edge radjus $R$, and focused down over a distance $F$ to a spot radius $r_{s}$ at the entrance to a self-pinched transport region. Then the minimum net current required to contain the beam in the self-pinched mode at radius $r_{c}$ occurs when $r_{c}=\sqrt{2} r_{s}$, and the current is $I_{n t t}=2\left(R / r_{c}\right)^{2} \theta_{\mu}{ }^{2} \beta \gamma(A / q) M^{3} / e$, where $\beta=v / c, v$ is the ion speed, $\mathrm{c}$ is the speed of light, $\gamma=\left(1-\beta^{2}\right)^{-1 / 2}, M$ is the mass of a proton, and $\mathrm{e}$ is the charge of an electron. For typical heavy ion parameters for IFE $(A=133, Z=55, q=55$, $\beta=0.2$, microdivergence $\left.\theta_{\mu}=0.3 \mathrm{mrad}, \mathrm{R}=15 \mathrm{~cm}, \mathrm{r}_{\mathrm{c}}=0.25 \mathrm{~cm}\right), \mathrm{I}_{\text {net }}=10 \mathrm{kA}$; which corresponds to a required net current fraction $\left(I_{\text {net }} / I_{e}\right)$ of only $1 \%$, for an $I_{e}$ of $1 \mathrm{MA}$. For a scaled proton experiment with a larger radius channel $\left(A=1, Z=1, q=1, \beta=0.056, \theta_{\mu}\right.$ $=20 \mathrm{mrad}_{,} R=10 \mathrm{~cm}, \mathrm{r}_{\mathrm{r}}=2.5 \mathrm{~cm}$ ), we obtain $I_{\text {net }}=25 \mathrm{kA}$, which corresponds to a net current fraction of $25 \%$ for an injected current of $100 \mathrm{kA}$. The important point is that both heavy ions and protons obey the same equation for $\mathrm{I}_{\text {net }}$ and that a proton self-pinched transport experiment could be used to benchmark a computer code such as IPROP. which then could be used to predict heavy ion self-pinched transport behavior for full IFE parameters.

Computer codes used in the late 1970's predicted self-pinched transport at about 1 Torr would not work because the net current would be too low. However, a new, hybrid, 3-D, EM code named IPROP ${ }^{3}$ has been developed that can accurately model charge and current neutralization for an ion beam injected into neutral gas, including non-local breakdown effects caused by energetic secondary electrons. IPROP uses PIC ions, PIC electrons for electron energies above some energy (typically $100 \mathrm{eV}$ ), and fluid electrons for lower energies. IPROP has already been-benchmarked against extensive gas breakdown experiments at NRL using proton beams injected into $\mathrm{He}, \mathrm{Ne}, \mathrm{Ar}_{\text {, }}$ and air ${ }^{3}$. The same code predicts that self-pinched transport should work within a useful range of gas pressures and beam parameters. Numerous IPROP simulations for proton beams and heavy ion beams predict that net current fractions up to $30 \%$ for protons and $50 \%$ for heavy ions will occur with an optimum gas pressure in the 1-100 mTorr regime ${ }^{4}$. A characteristic trumpet-shaped, beam-front envelope forms and propagates. Proton experiments are now 
needed to compare with IPROP's predictions, and validate IPROP's credibility for scaling to full heavy ion fusion IFE parameters.

A successful proton experiment would be the first demonstration of self-pinched transport with an ion beam. Electron beams in the 1 Tor regime have already been routinely propagated in the self-pinched regime with substantial net currents for the past two decades. However, gas breakdown produced by ion beams is substantially different from electron-beam produced gas breakdown, and no ion beam self-pinched transport experiments have yet been attempted. Other issues include beam head erosion effects, possible instabilities (hose, two-stream, filamentation), and guiding/tracking effects. All of these issues have been considered theoretically to some degree, although further theoretical study and experiments are still needed.

\section{Suggested Near Term Experiments and Analysis}

At the present time, an opportunity exists to carry out experiments to demonstrate self-pinched transport of an intense ion beam in the laboratory. The required high-current, high-quality ion beam has been developed on the Gamble II pulsed-power generator at the NavaI Research Laboratory (NRL). A new applied magnetic-field diode has been fielded recently on this generator. The new diode is the first shallow-focusing, large-area diode designed in extraction geometry for generating and focusing a high-current ion beam appropriate for small radius transport experiments. A 1.3 to $1.5-\mathrm{MeV}, 100-\mathrm{kA}$ proton beam with a microdivergence $\theta_{\mu}$ of 20 to $30 \mathrm{mrad}$ has been produced and focused to a few $\mathrm{cm}$ radius at $70 \mathrm{~cm}$ from the diode. IPROP simulations indicate that this beam is appropriate for testing the self-pinched transport concept. No other accelerator can provide this high-current focused-beam capability at this time.

The Pulsed Power Physics Branch at NRL has extensive experience in ion-beam generation and transport. Intense ion beams of large microdivergence ( $200 \mathrm{mrad}$ ) were first produced in the late 1970's on Gamble II at NRL using a pinch-reflex diode. Beams from this diode have been transported up to several meters for a variety of applications, including evaluation of beam transport and focusing schemes for light-ion inertial-confinement-fusion, studies of gas breakdown and ionization by intense proton beams, and simulation of nuclear weapon effects. This work has included extensive experimental and theoretical investigations of ballistic transport and focusing, Z-discharge transport, and wire-guided transport. IPROP simulations carried out within the group during the past three years have provided the theoretical basis for designing self-pinched transport experiments using the recently developed proton beam capability. These simulations predict that sufficient net current for self-pinching can be established with the beam conditions already achieved with the new applied-B diode on Gamble II. The expertise and experience from these efforts is available now to be applied to self-pinched transport experiments.

This research group has also developed an wide array of ion-beam diagnostics in the course of this work. Beam intensities, spatial and temporal profiles, beam composition, energy spectra, and net currents of partially neutralized beams have all been measured. A new diagnostic based on the Zeeman effect is being developed for beam transport studies in low-pressure gases and will be available within the next few months. This technique measures the magnetic freld evolution within the beam envelope with radial resolution and can be used to infer net current distributions within the beam. Laser interferometry has been developed and used to time resolve the electron density evolution within an ion beam propagating in a background gas.

This demonstrated technical capability can now be used to test the feasibility of self-pinched transport for HIF. Using the focused-beam conditions recently developed at NRL, experiments can be designed immediately to evaluate transport of protons in a background gas (either He or Ar) over a range of pressures appropriate for self-pinched 
transport. Measurements of the transport efficiency (using nuclear activation) and the total net current in the beam (using the recently developed Zeeman diagnostic) can be used to determine the optimum pressure for transport. Beam dynamics during transport can be stadied using time-resolved Rutherford-scattered images of the beam radial profile. Erosion of the predicted trumpet-shaped beam head during transport can be inferred by identifying where and when protons are lost to the transport channel walls using radiachromic film and/or Faraday cups. Interferometric measurement of the electron density can be used to study ionization of the background gas.

Results of these experiments can be analyzed with additional IPROP calculations which simulate the actual experimental conditions. IPROP can predict gas electron production, beam dynamics, net currents, and transport efficiencies. Comparison of these quantities with measurements can validate the IPROP code for ion-beam transport studies. The success of this program will be determined by the observation of beam self-pinching, by the efficiency of proton transport to the end of the channel, and by the degree to which the code results agree with experiment. An experimental demonstration of efficient self-pinched transport and agreement between measurements and IPROP results will be a major achievement for the HIF program. The IPROP code can then be used with greater confidence to evaluate self-pinched transport conditions for HIF power plant designs.

\section{Conclusion}

A number of HIF IFE final transport schemes have been discussed. Self-pinched transport has a high payoff in regard to its advantages in an HIF power plant scenario. An opportunity now exists to experimentally demonstrate self-pinched transport of ions for the first time with a high-current proton beam from the Gamble II accelerator at NRL. It is recommended that OFES support this development. 


\section{APPENDIX}

\section{Selected References:}

\section{Ion Beam Transport and Stability}

"Ion Beam Propagation and Focusing," C. L. Olson, J. Fusion Energy 1, 309 (1982).

"Filamentation of a Heavy Ion Beam in a Reactor Vessel", E. P. Lee, S. Yu, H. L. Buchanan, F. W. Chambers, and M. N. Rosenbluth, Phys. Fluids 23, 2095 (1980).

"Z-Discharge Transport of Intense Ion Beams for Inertial Confinement Fusion," P. F. Ottinger, D. V. Rose, D. Mosher, and J.M. Neri, J. Appl. Phys. 70, 5292 (1991).

"Chamber Propagation Physics for Heavy Ion Fusion," D. A. Callahan, Fusion Engineering and Design $\underline{32-33}, 441$ (1996).

\section{Gas Breakdown Experiments}

"Current Neutralization of Intense MeV Proton Beams Transported in Low-Pressure Neutral Gas", F. C. Young, D. D. Hinshelwood, R. F. Hubbard, M. Lampe, J. M. Neri, C. L. Olson, P. F. Ottinger, R. V. Rose, S. P. Slinker, S. J. Stephanakis, and D. R. Welch, Phys. Rev. Lett. 70,2573 (1993).

"Interaction of Intense MeV Light Ion Beams with Low-Pressure Gasses," F. C. Young, R. F. Hubbard, M. Lampe, J. M. Neri, P. F. Ottinger, S. J. Stephanakis, S. P. Slinker, D. D. Hinshelwood, D. V. Rose, C. L. Olson, D. R. Welch, Phys. Plasmas 1,1700 (1994).

\section{IPROP Code}

"Simulations of Charged-Particle Beam Transport in a Gas Using a Hybrid Particle-Fluid Plasma Model," D. R. Welch, C. L. Olson, and T.W. L. Sanford, Phys. Plasmas 1, 764 (1994).

\section{Self-Pinched Transport}

"Self-Pinched Transport for Ion Beam Driven Inertial Confinement Fusion," C. L. Olson, D. L. Hanson, M. E. Cuneo, T. R. Lockner, P. R. Menge, J. E. Bailey, A. B. Filuk, J. W. Poukey, I. R. Shokair, B. M. Marder, S. A. Slutz, J. P. Quintenz, P. F. Ottinger, F. C. Young, D. D. Hinshelwood, T. G. Jones, D. Mosher, J. M. Neri, B. V. Oliver, D. V. Rose, S. J. Stephanakis, E. P. Lee, K. D. Hahn, S. S, Yu, R. O. Bangerter, D. R. Welch, A. Friedman, A. B. Langdon, B. G. Logan, P. Hoppe, H. Bluhm, A. Nakagawa, R. R. Peterson, J. J. MacFarlane, P. Wang, and G. L. Kulcinski, International Atomic Energy Agency Sixteenth IAEA Fusion Energy Conference (Montreal, Canada, October 711, 1996) IAEA-F1-CN-64/BP-14.

"Self-Pinched Transport for Ion-Driven Inertial Confinement Fusion," D. R. Welch and C. L. Olson, Fusion Engineering and Design 32-33, 477 (1996).

"A Study of Stripped Pinched-Beam Transport for Heavy Ion Fusion," K. Hahn and E. Lee, Fusion Engineering and Design, 32-33, 417 (1996). 


\section{IFE Power Plant Studies Using SeIf-Pinched Transport}

"LIBRA-SP, A Self Consistent Design of a Commercial Fusion Power Plant Based on Self-Pinched Propagation of Ions," B. Badger, D. Bruggink, P. Cousseau, R. L. Engelstad, Y.-M. Lee, H. Y. Khater, G. L. Kulcinski, J. J. MacFarlane, E. A. Mogahed, G. A. Moses, R. R. Peterson, S. Rutledge, M. E. Sawan, I. N. Sviatoslavsky, P. Wang, L. J. Wittenberg, C. L. Olson, and R. E. Olson, Fusion Power Associates Report FPA94-6 (December, 1994).

"Inertial Fusion Energy, Reactor Design Studies, Prometheus - H," McDonnell Douglas Aerospace Team, Final Report, Volumes I-III, DOE/ER 54101, MDC 92E0008 (March, 1992).

"Liquid Wall Inertial Fusion Energy Power Plants," R. Moir, Fusion Engineering and Design, 32-33, 93 (1996).

\section{Related Efforts:}

Several USA government laboratories, universities, and companies have worked on areas related to the proposed self-pinched transport experiments. A brief description of each research activity is as follows:

LBNL Research on final focus and transport in the reactor chamber with present focus on ballistic transport; emphasis on Z-discharge channel transport and selfpinched transport as an attractive option. Center for the U.S. Heavy Ion Fusion Program.

LLNL Computer simulation of beam dynamics during final focus and chargeneutralized final transport of heavy ion beams.

NRL Experimental and theoretical research on all beam transport methods for high-current, low-atomic-number ion beams. Present emphasis directed towards a self-pinched transport demonstration experiment.

SNL Research on generation and focusing of high-current low-atomicnumber ion beams, and theoretical research on beam transport including self-pinched transport. This program, funded by US DOE-DP, is being closed out during FY98.

MRC Theory and computer simulations using IPROP of self-pinched transport for proton and heavy-ion parameters.

U. Wisconsin IFE power plant design for ion beam ICF with special emphasis on the advantages of self-pinched transport. 